\title{
Experimental Studies on Homologous Heart Transplantation
}

\section{Second Report: Pharmacological Studies on the Transplanted Heart}

By

Fumio Jin

\author{
From the Surgical Clinic of Prof. S.T. Katsura, Faculty \\ of Medicine, Tohoku University, Sendai
}

(Received for publication, November 25, 1959)

Though isolated heart preparations have been widely used for study of physiology and pharmacology of the heart, the heart transplantation was suggested as a new device for such purposes by Mann and his associates ${ }^{1)}$ in 1933. They studied the pharmacological effect of thyroxin on the transplanted heart by using the cervical canine heart transplantation which was a first invention in this field. Since their excellent report, there is almost no communication in the literature except a study of Marcus et al. ${ }^{2)}$

As a technical device for study of the heart, the heart tansplantation would likely have some advantages over the isolated heart preparation; the denervated heart's functions are easily observed in comparison with the activity of normal heart in the same animal's body.

With such a fact in mind, the author of this paper undertook the following experiments, for the purpose of studying the behavior of the denervated heart in comparison with the normal heart in the same animal's body against various kinds of drugs.

\section{METHOD}

Mongrel dogs were used for the experiments and the heart was transplanted in the same manner as described in Report $\mathbf{I}$. After comfirming that the heart rate of the transplant was stable by ECG record following endotracheal ether anesthesia induced in the recipient dog, the below mentioned drugs were injected intravenously from the leg. All drugs were diluted with $5 \%$ glucose solution to the volume of $5 \mathrm{cc}$ and injected at rate of $2.0 \mathrm{cc}$ per minute. Following the injection, the V-lead of ECG was taken at 30 seconds, intervals for the first 5 minutes, at 2 minutes' intervals thereafter and the cardiac rate of the transplant and the reciplient's heart were recorded.

神文堆 
The drugs employed in this experiments were as follows.

1. Vasopressants

Epinephrine : 0.0034 and $0.028 \mathrm{mg}$. per $\mathrm{kg}$. of the recipient's weight.

Nor-adrenaline: 0.0055 and $0.004 \mathrm{mg}$. per $\mathrm{kg}$.

Neosynephrine: 0.022 and $0.01 \mathrm{mg}$. per $\mathrm{kg}$.

Ephedrine: 2.3 and $1.5 \mathrm{mg}$. per $\mathrm{kg}$.

2. Central nerve stimulants

Cardiazol (Pentamethylenetetrazol) : $0.015 \mathrm{mg}$. and $0.01 \mathrm{mg}$. per $\mathrm{kg}$.

Aminocordine (Coramine): $18 \mathrm{mg}$. per $\mathrm{kg}$.

3. Drugs acting on the parasympathetic nervous system

Atropine : 0.038 and $0.05 \mathrm{mg}$. per $\mathrm{kg}$.

Pilocarpine: 0.6 and $0.3 \mathrm{mg}$. per $\mathrm{kg}$.

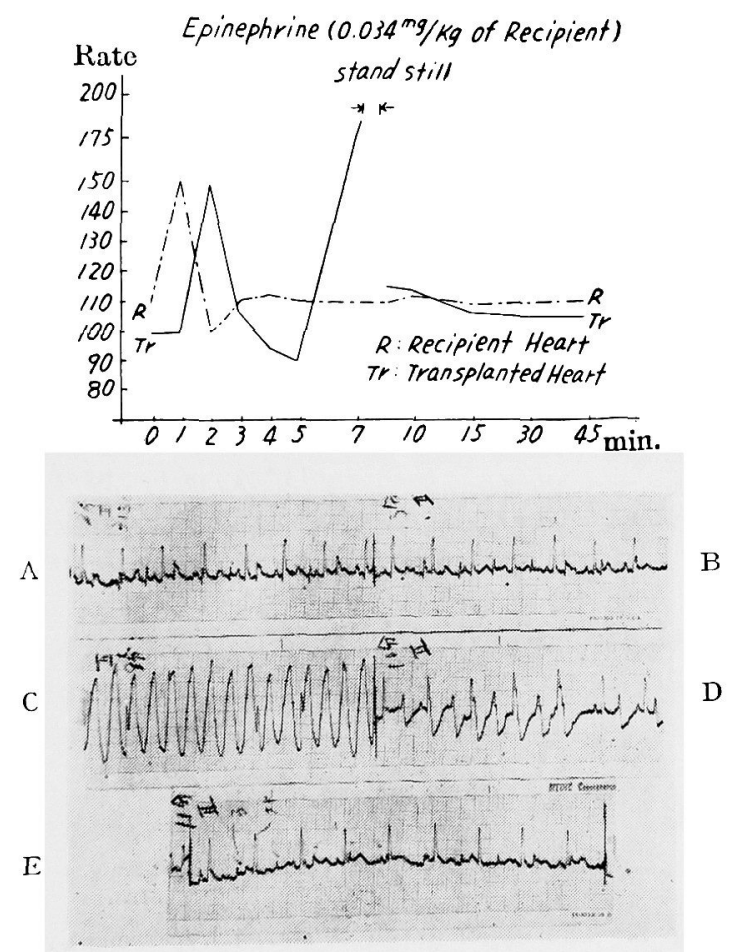

Fig. 1. The ECG of V-lead was traced simultaneously with the above graph of transplanted cardiac rates. A : 2 min. after injection. B : 3 min. after injection. C : Ventricular fibrillation of transplanted heart at 7 min. after injection. D:Transplanted heart starts again to beat after systolic cardiac standstill. $\mathbf{E}$ : ECG of transplant at $11 \mathrm{~min}$. after injection is nearly the same as before injection. The small deflections of ECG are the transplant's and the large complexes are the recipient's. 


\section{RESULTS}

Vasopressants: Epinephrine especially showed a distinguished pattern in its action on the rate of both hearts. Immediately following the injection the rate became rapid in one to two minutes, then a counteractive bradycardia developed and again the cardiac rates were increased. To a large amount of the drug, the transplanted heart reacted more intensively than the recipient heart and finally a systolic cardiac standstill ensued from ventricular tachycardia of the transplanted heart (Figs. $1 \& 2$ A).

Nor-adrenaline coused tachycardia in both the hearts for a period of one hour. The transplanted heart sometimes showed temporarily slow cardiac rates two to four minutes after the injection (Fig. 2 B).

A

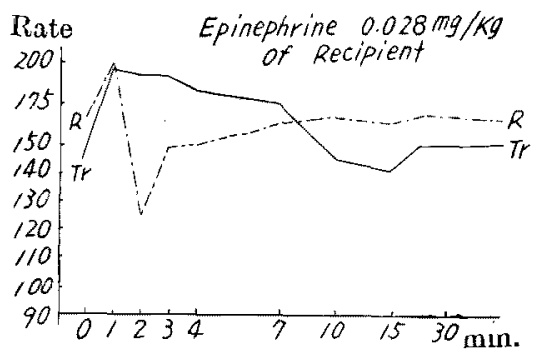

B

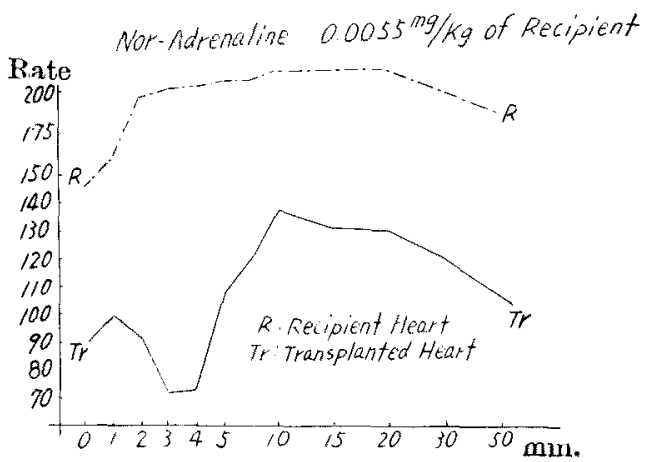

Fig. 2. The upper graph A shows hypersensitivity of transplant to relative small amount of epinephrine. The lower graph B shows the accelerating effect of nor-adrenaline.

In the group given neosynephrine, the cardiac rate in both the hearts was reduced to about 10 to 20 per minute without exception. (Fig. 3 A).

Ephedrine acted as an accelerator on both the hearts but its action on the transplant was stronger than on the recipient heart (Fig. $3 \mathrm{~B}$ ). 
$A$

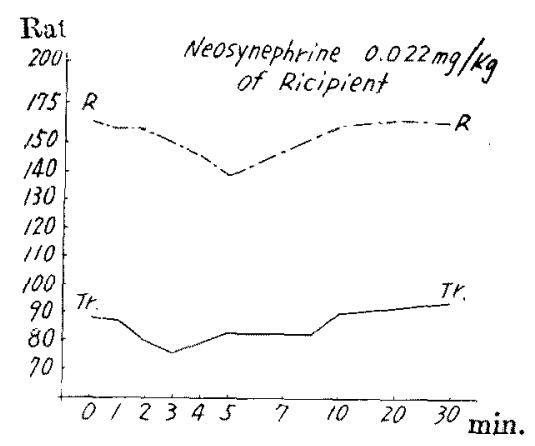

B

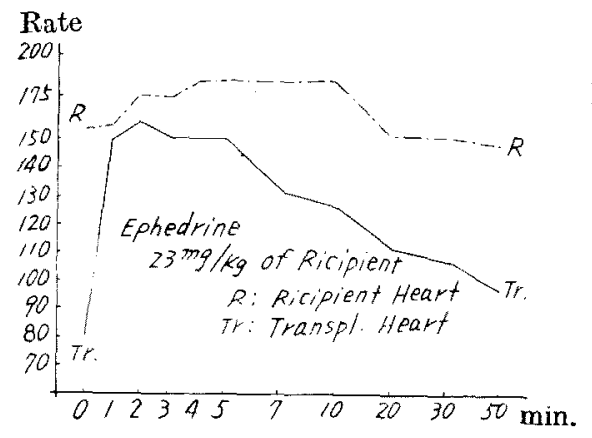

C

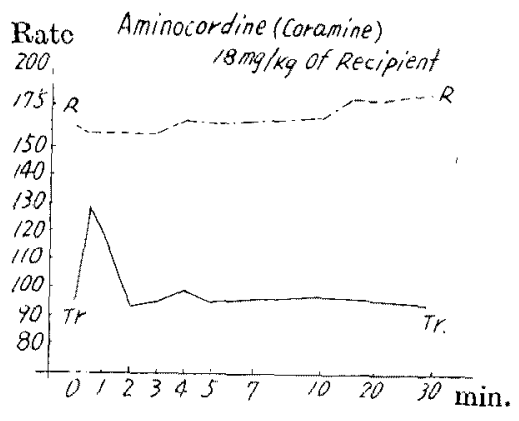

$\mathrm{D}$

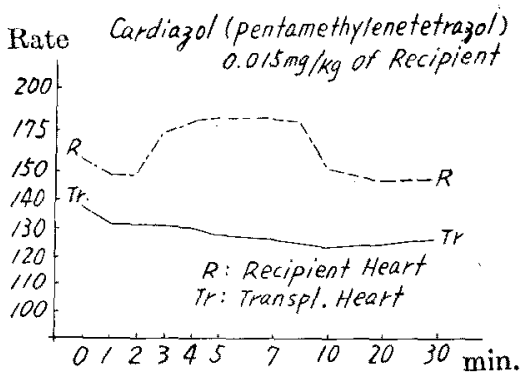

Fig. 3. A : neosynephrine causes bradycardia in both recipient and transplanted hearts. B : transplanted heart showed hypersensitivity to ephedrine. $\mathrm{C}$ : the initial tachycardia by aminocordine in transplanted heart is probably due to lightening of anesthesia. D : cardiazol causes no remarkable effect on the rate of transplanted heart.

Central nerve stimulants: With both cardiazol and coramine, the cardiac rates were not changed remarkably (Fig. $3 \mathrm{~A} \& \mathrm{~B}$ ). Increased cardiac rates with the transplanted heart after coramine in Fig. $3 \mathrm{C}$ was likely due to lightening of the anesthesia.

Parasympathetic drugs: Though a large amount of atropine induced slightly rapid cardiac rate, pilocarpine displayed an intense action on the recipient heart (Fig. 4 A). After injection of pilocarpine, the recipient heart rapidly slowed down in about 3 minutes and finally developed cardiac standstill, whereas the transplanted heart showed slightly slow cardiac rate even when the recipient heart was in cardiac standstill (Fig. 4 B).

\section{DISCUSSION}

From these experiments, it is found that the heart transplantation is a very good method for study of the physiology or pharmacology of the denervated, 


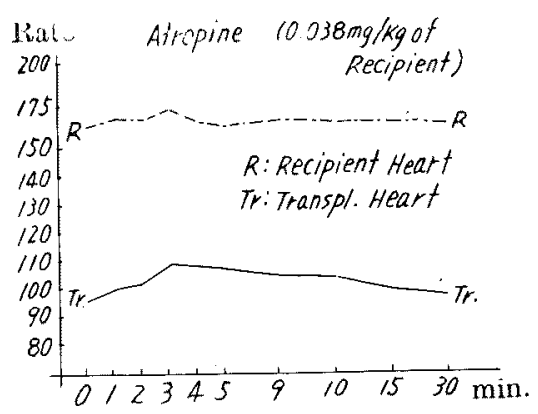

B

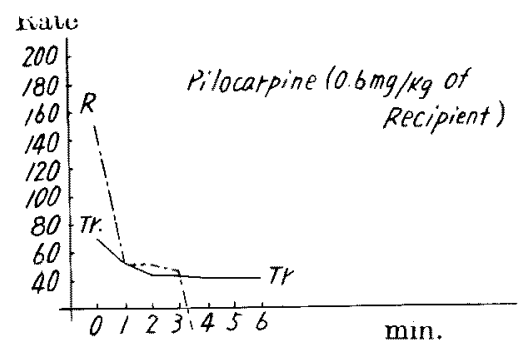

Fig. 4. The upper groph shows the effect of atropine : slight tachycardia in both hearts. The lower shows the effect of pilocarpine : recipient heart showed cardiac standstill after intensified bradycardia at $3 \mathrm{~min}$. while transplanted heart is keeping a proper beat.

namely, transplanted heart in comparison with the normal recipient heart's action in the same body. Pharmacological study of the transplanted heart has interested only few investigators and we find only two communications: Mann and his associates reported on thyroxin and Marcus and his coworkers studied on digitalis or epinephrine without detailed protocol. For many years it has been known that certain organs became more sensitive to drugs when deprived of their nerve supply. ${ }^{31,4)}$ With such previous reports in my mind, the problem of the supersensitivity of the denervated heart was studied. With epinephrine the denervated heart showed a very intense reaction in comparison with the recipient heart and in large dosis the transplanted heart suffered a systolic cardiac standstill, whereas the recipient heart showed a regular biphasic epinephrine reaction. In this experiment, the sensitivity of denervated heart is high in the order of epinephrine, ephedrine and nor-adrenaline. It is interesting that nor-adrenaline shows slight tachycardia in both the hearts, whereas bradycardia is usually induced by its clinical application. Bradycardia following neosynephrine injection is said to be due to vagal stimulation ${ }^{5}$, but in this experiment the drug showed some 
- direct myocardial action so far as bradycardia of the denervated heart is concerned. Before making the tests with central nerve stimulants the author expected something from its actions because the transplanted heart easily inclined to a tachycardia following a light exercise or excitement of the recipient dog. Neither aminocordine nor cardiazol showed any perceptible effect on the hearts. Sympathomimetic drugs mostly induced sensitivity of the denervated heart, but to parasympathetic drugs the denervated heart showed strong resistance. These contradictory facts on the sensitivity of the denervated heart may need further study in future.

\section{SUMMARY}

The cervical heart transplantation is a new and promising device for study of pharmacology of the denervated heart. In this experiments, pharmacological studies of vasopressants, central nervous stimulants and parasympathetic drugs on the denervated heart are studied in comparison with the recipient heart in the same body.

1. As a technique for investigating the denervated heart, the usefulness of the cervical heart transplantation described by Mann, Priestley, Marcowitz and Yater was proved.

2. Highsensitivity of the denervated heart to sympathomimetic drugs was found to be in the order of epinephrine, ephedrine and nor-adrenaline.

3. On the contrary, parasympatheties evoked no highsensitivity of the denervated heart.

4. Central nerve stimulants have no action on the denervated heart.

\section{References}

1) Mann, Priestley, Marcowitz \& Yater, Arch. Surg., 1933, 26, 219.

2) Marcus, Wong \& Luisada, ibid, 1953, 66, 179.

3) Elliott, J. Physiol., 1905, 32, 401.

4) Ray \& Console, J. Neurosurg., 1948, 5, 23.

5) Keys \& Violante, J. Clin. Invest., 1942, $21,1$. 